\title{
Multiple ring nebulae around blue supergiants ${ }^{\star}$
}

\author{
S. M. Chiţă ${ }^{1}$, N. Langer ${ }^{1}$, A. J. van Marle ${ }^{1,2}$, G. García-Segura ${ }^{3}$, and A. Heger ${ }^{4,5}$ \\ 1 Astronomical Institute, Utrecht University, PO Box 80000, 3508 TA Utrecht, The Netherlands \\ e-mail: s.m.chita@uu.nl \\ 2 Bartol Research Institute, University of Delaware, 102 Sharp laboratory, Newark, 19716 DE, USA \\ 3 Instituto de Astronomía - UNAM, APDO Postal 877, Ensenada, 22800 Baja California, Mexico \\ 4 Theoretical Astrophysics Group, T-6, MS B227 Los Alamos National Laboratory, Los Alamos NM 87545, USA \\ 5 School of Physics and Astronomy, 112 Church st, University of Minnesota, Minneapolis, MN 55455, USA
}

Received 29 April 2008 / Accepted 16 July 2008

\section{ABSTRACT}

\begin{abstract}
Context. In the course of the life of a massive star, wind-wind interaction can give rise to the formation of circumstellar nebulae which are both predicted and observed in nature.

Aims. We present generic model calculations to predict the properties of such nebulae for blue supergiants.

Methods. From stellar evolution calculations including rotation, we obtain the time dependence of the stellar wind properties and of the stellar radiation field. These are used as input for hydro-calculations of the circumstellar medium throughout the star's life.

Results. Here, we present the results for a rapidly rotating $12 M_{\odot}$ single star. This star forms a blue loop during its post main sequence evolution, at the onset of which its contraction spins it up close to critical rotation. Due to the consequent anisotropic mass loss, the blue supergiant wind sweeps up the preceding slow wind into an hourglass structure. Its collision with the previously formed spherical red supergiant wind shell forms a short-lived luminous nebula consisting of two polar caps and a central inner ring. With time, the polar caps evolve into mid-latitude rings which gradually move toward the equatorial plane while the central ring fades. These structures are reminiscent of the observed nebulae around the blue supergiant Sher 25 and the supernova 1987A.

Conclusions. The simple model of an hourglass colliding with a spherical shell reproduces most of the intriguing nebula geometries discovered around blue supergiants, and suggests that they form an evolutionary sequence. Our results indicate that a binary system is not required to obtain them.
\end{abstract}

Key words. hydrodynamics - ISM: bubbles - stars: winds, outflows - stars: supergiants

\section{Introduction}

During the course of their evolution, massive stars have strong winds which eject matter into their surroundings. During their post-main sequence evolution, these stars can move back and forth between the blue and the red side of the Hertzsprung-Russell (HR) diagram, with little time spent at intermediate effective temperatures (e.g., Langer 1991b). Hydrodynamic considerations imply that each such transition produces a circumstellar shell: when the star moves from the blue to the red side of the HR diagram, the slow red supergiant (RSG) wind will be stalled by the high pressure of the previously created hot wind bubble, and will accumulate into a shell at the location where this pressure equals the RSG wind ram pressure (García-Segura et al. 1996b). We call such a more or less stationary shell the RSG shell. When the star moves from the red to the blue side of the HR diagram, the wind speed increases and the blue supergiant (BSG) wind plows up the preceding RSG wind into a rapidly expanding shell, which we call the BSG shell.

Consequently, we expect a spectacular circumstellar phenomenon for stars undergoing so called blue loops, namely that it triggers the formation of an expanding BSG shell, which will at some point collide with the previously formed stationary RSG shell. While both the RSG and the BSG shell by itself

\footnotetext{
* Appendix A is only available in electronic form at http://www . aanda.org
}

may be difficult to observe, their violent interaction may release enough energy to provide an observable nebula.

Despite this simple and intriguing expectation, there are so far only few attempts to obtain quantitative prediction for the outcome of the described shell interaction (see Blondin et al. 1993; Martin et al. 1995; Podsiadlowski et al. 2005). Within an effort to describe this phenomenon through generic calculations, which use detailed stellar evolution models as input for the circumstellar hydrodynamic modeling (Chiţă et. al., in preparation), we focus here on the results for a rotating $12 M_{\odot}$ single star.

\section{Computational method}

As input for our circumstellar hydrodynamic calculations we use the results of a stellar evolution calculation for a star of $12 M_{\odot}$ and a metallicity of $Z=0.02$. Specifically, we utilize Model F12B from Heger \& Langer (2000), which has an initial rotational velocity of $328 \mathrm{~km} \mathrm{~s}^{-1}$. The code used to compute this model includes OPAL opacities, detailed nuclear networks, mass loss according to Nieuwenhuijzen \& Jager (1990), the physics of rotation for the stellar interior, and rotationally modulated stellar wind, as described in Heger et al. (2000).

The evolution of the stellar model in the HR diagram is show in Fig. A.1. At core-H exhaustion, it moves to the RSG regime where it remains for $825000 \mathrm{yr}(\sim 60 \%$ of the core-He burning 
Table 1. Ejected mass $(\Delta M)$, momentum $(\Delta p)$ and kinetic energy $(\Delta E)$ during the various evolutionary phases of our stellar model.

\begin{tabular}{ccccc}
\hline \hline Phase & $\Delta t$ & $\Delta M$ & $\Delta p$ & $\Delta E$ \\
& $10^{3} \mathrm{yr}$ & $M_{\odot}$ & $10^{38} \mathrm{~g} \mathrm{~cm} \mathrm{~s}^{-1}$ & $10^{45} \mathrm{erg}$ \\
\hline MS & 19200 & 0.43 & 396 & 1480 \\
RSG I & 825 & 0.33 & 71 & 38 \\
RR & 25 & 0.02 & 7.2 & 6.0 \\
BSG & 550 & 0.11 & 52 & 68 \\
RSG II & 225 & 0.13 & 25 & 12 \\
\hline
\end{tabular}

The evolutionary phase is identified in the first column: main sequence phase (MS), first red supergiant phase (RSGI), phase of rapid rotation (RR), blue supergiant stage (BSG), and second red supergiant phase (RSGII). The approximate duration of each phase is given in the second column.

life time), before it undergoes a blue loop. It then stays in the BSG regime of the HR diagram for the remaining period of core helium burning before it moves back to the RSG regime where it explodes as a type II supernova.

As shown by Heger \& Langer (1998), as the convective envelope retreats during the onset of the blue loop, all its angular momentum is concentrated in a small amount of mass in the top layers of the star by the time that convection vanishes. Blue loops therefore provide a natural way to bring the stellar surface to close to critical rotation. This also happens in our chosen stellar model (Fig. A.2). The limit of critical rotation is reached during the red-blue transition, which produces a brief period of strong, slow and anisotropic mass loss (Table 1). The strong mass loss then reduces the rotation rate of the stellar surface (Langer 1998), and the star settles at a rotation velocity of about $50 \mathrm{~km} \mathrm{~s}^{-1}$ in the BSG regime.

To simulate the evolution of the circumstellar matter (CSM), we use the ZEUS 3D code developed by Stone \& Norman (1992) and Clark (1996). ZEUS 3D is an explicit non-conservative code that solves the hydrodynamic equations as partial, finite difference equations on a fixed, staggered mesh grid. Radiatively optically-thin cooling is included by solving the energy equation implicitly according to Mac Low et al. (1989), and by using the plasma cooling curve of MacDonald \& Bailey (1981). We compute the evolution of the CSM during the main sequence and the early RSG stage in 1D, with 4500 grid points over a radius of $45 \mathrm{pc}$, and we assume an interstellar medium density of $1 \mathrm{~cm}^{-3}$. After $100000 \mathrm{yr}$ into the first RSG stage, we map the 1D model onto a $2 \mathrm{D}$ spherical grid to compute its further evolution. The inflow inner boundary condition is applied at $0.025 \mathrm{pc}$, and the outer boundary remains at $45 \mathrm{pc}$. The radial component of the grid is resolved with 1000 grid points, where 900 grid points are used for the inner $5 \mathrm{pc}$, and 100 grid points for the outer $40 \mathrm{pc}$. The angular coordinate of 90 degrees is resolved with 200 grid points. The method used here was used before by García-Segura \& Franco (1996), García-Segura et al. (1996a,b) and van Marle et al. $(2005,2007)$.

We are using the time dependent mass loss rate and the terminal wind speed from the stellar evolution model as input in our central mesh point for the hydrodynamic calculations. The wind speed is obtained from the stellar escape velocity using the scaling proposed by Eldridge (2005). The wind anisotropy is described using the equations of Bjorkman \& Cassinelli (1993), as in Langer et al. (1999). For near-critically rotating stars, this provides a slow and dense equatorial outflow, and a fast wind in polar directions. We note that while the Bjorkman-Cassinelli mechanism has been criticized in the context of line driven winds
(Owocki et al. 1996), it is unclear whether line driving does play a major role in the situation of near-critical rotation.

The effect of photoionization was included in the simulations by calculating the Strömgren radius along each radial grid line as described in García-Segura et al. (1999) and van Marle et al. $(2005,2007,2008)$. The number of ionizing photons is computed according to the effective temperatures and surface gravities of the stellar evolution model, by interpolating in a grid of model atmospheres for massive OB stars of solar metallicity computed with the FASTWIND non-LTE code (Puls et al. 2005) as described in Lefever et al. (2007). and

\section{Results}

During its main sequence phase our $12 M_{\odot}$ star creates a hot bubble in the interstellar medium which, at core hydrogen exhaustion, is characterized by a radius of $30 \mathrm{pc}$ and an internal energy density of $10^{-12} \mathrm{erg} \mathrm{cm}^{-3}$. Once the star has become an RSG, a slow ( $\left.\sim 50 \mathrm{~km} \mathrm{~s}^{-1}\right)$, dense and isotropic wind is injected into the computational domain (Fig. A.2). This RSG wind accumulates at a distance of $\sim 1.5 \mathrm{pc}$ where its ram pressure is balanced by the hot bubble pressure, and forms an RSG shell (cf. García-Segura et al. 1996b). At the end of the first RSG phase, this shell contains about $0.26 M_{\odot}$. It is rather extended ( $\Delta r \simeq 1 \mathrm{pc})$, and its central parts have condensed due to cooling.

At the onset of the blue loop, the central star reaches closeto-critical rotation, and ejects a dense equatorial disk (Heger \& Langer 1998). While mass and time scales differ, this phenomenon occurs analogously to the simulation of the outburst of $\eta$ Carinae by Langer et al. (1999). As in this case, the ensuing BSG wind sweeps up the preceding slow wind material into an "hourglass" structure (Fig. A.4). On a time scale of a few $10^{4} \mathrm{yr}$, this hourglass expands into the sphere defined by the RSG shell, with a maximum velocity of $\sim 130 \mathrm{~km} \mathrm{~s}^{-1}$ (Fig. A.3). The faster polar parts of the hour glass hit the inner edge of the RSG shell first. The collision creates a hot $\left(T \simeq 10^{5} \mathrm{~K}\right)$ and dense $\left(n \simeq 10 \mathrm{~cm}^{-3}\right)$ pair of polar caps. As time proceeds, the collision zone moves to lower latitudes of the RSG shell and becomes more confined in latitude. At the same time, the interaction of the BSG wind with the equatorial disk defines a second, ring-shaped collision zone in the equatorial plane, which expands over time with a velocity of $18 \mathrm{~km} \mathrm{~s}^{-1}$.

Figure 1 shows snapshots of the emissivity map, according to the cooling curve employed in our hydro simulations, for three moments in time, along with projection maps constructed from rotationally symmetric 3D-structures obtained from the 2D maps. Here, only emission from gas in the temperature range between $10^{4} \mathrm{~K}$ and $10^{6} \mathrm{~K}$ is considered, which is the dominant component. Hotter gas, which is formed from the reverse shock of the collision, might be observable in the X-ray regime; the peak luminosity of this component in our model is $10^{33} \mathrm{erg} \mathrm{s}^{-1}$, which is achieved about $50000 \mathrm{yr}$ after the onset of the collision. At an early interaction stage, the radiation is dominated by two polar caps and one equatorial ring, later on by two mid-latitude rings and one fading smaller equatorial ring, and finally two midlatitude rings at rather low latitude are visible. These two rings gradually move to the equatorial plane while fading. The full time dependence of the emission structure is shown in an accompanying film clip which is available on the A\&A website.

The energy budget for the collision of the polar caps of the hour glass with the RSG shell follows directly from the stellar properties. The polar caps have an emissivity of $l \simeq 10^{-21} \mathrm{erg} \mathrm{cm}^{-3} \mathrm{~s}^{-1}$ in a volume of $V \simeq 4 \pi r^{2} \Delta r=4 \times 10^{54} \mathrm{~cm}^{3}$ 

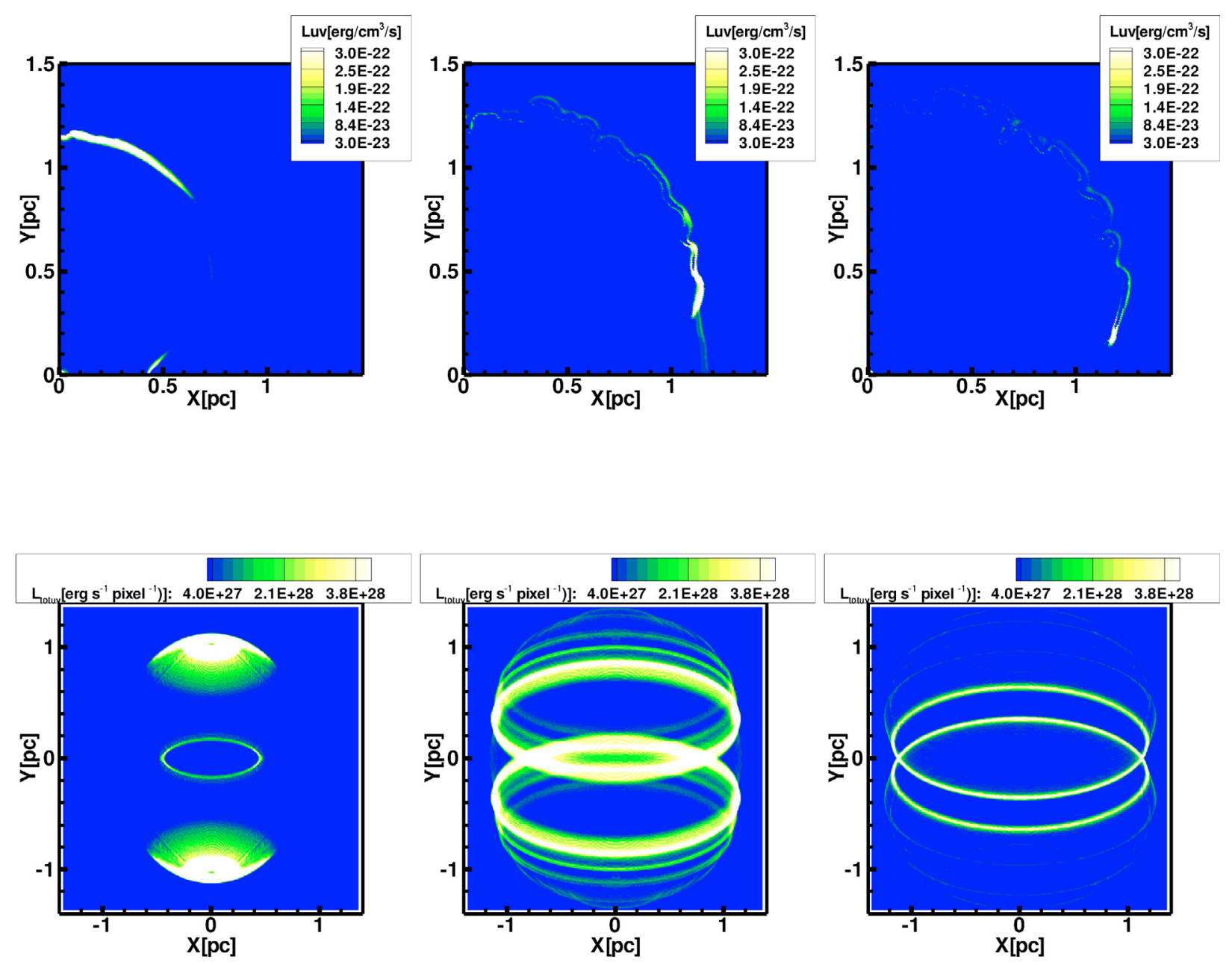

Fig. 1. Emission structures from our 2D hydro simulation, for the same moments in time as panels 2 to 4 of Fig. A.4, i.e., $9000 \mathrm{yr}, 15000 \mathrm{yr}$, and $18000 \mathrm{yr}$ after the onset of the BSG wind. Upper panel: emissivity of the gas with $10^{4}<T<10^{6} \mathrm{~K}$, in the simulation plane. Lower panel: projections of the $3 \mathrm{D}$ emission obtained by assuming rotational symmetry of the $2 \mathrm{D}$ structures of the upper panel, viewed with an inclination angle of $60^{\circ}$, constructed with a resolution of $400 \times 400$ pixels.

(with $r=0.5 \mathrm{pc}$ and $\Delta r=0.04 \mathrm{pc}$; see Fig. 1). Thus, they shine with a total luminosity of $4 \times 10^{33} \mathrm{erg} \mathrm{s}^{-1}$, i.e. roughly one solar luminosity, with a time scale of $\tau_{\text {rad }}=l / u \simeq 9000 \mathrm{yr}$, where $u=\frac{3}{2} n k T$ is the internal energy of the gas, and $T \simeq$ $10^{5} \mathrm{~K}$ and $n \simeq 13 \mathrm{~cm}^{-3}$ (corresponding to $\rho \simeq 10^{-23} \mathrm{~g} \mathrm{~cm}^{-3}$; Fig. A.4). The total radiated energy of the polar caps is about $E_{\text {rad }} \simeq \tau_{\text {rad }} L \simeq 10^{45} \mathrm{erg}$. This corresponds well to the kinetic energy released due to the braking of the polar caps, which reach their maximum velocity of $v \simeq 130 \mathrm{~km} \mathrm{~s}^{-1}$ at the time of collision, where it is reduced to $v \simeq 50 \mathrm{~km} \mathrm{~s}^{-1}$ (Fig. A.3). That is, $\Delta E_{\text {kin }}=\frac{1}{2} \Delta M \Delta v^{2} \simeq 8 \times 10^{44} \mathrm{erg}$, with $\Delta M \simeq 1.2 \times 10^{-2} M_{\odot}$ and $\Delta v \simeq 80 \mathrm{~km} \mathrm{~s}^{-1}$. This kinetic energy can be compared with the BSG wind kinetic energy, which, for $\dot{M} \simeq 10^{-6.8} M_{\odot} \mathrm{yr}^{-1}$ and $v_{\text {wind }} \simeq 300 \mathrm{~km} \mathrm{~s}^{-1}$ (Fig. A.2), yields $\sim 1.2 \times 10^{45} \mathrm{erg}$ over a time period of $9000 \mathrm{yr}$. Thus, the polar caps shine because the hourglass shaped BSG shell collides with the spherical RSG shell.

A similar consideration could be made for the inner ring, which is produced by the collision of the BSG wind with the equatorial disk ejected by the central star during the phase of near critical rotation. The disk properties depend on the wind properties of the star during this phase. However, in particular their latitude dependence cannot be expected to be reliably predicted within the current assumptions. The total mass of the disk is determined by the mass loss of the star at critical rotation.
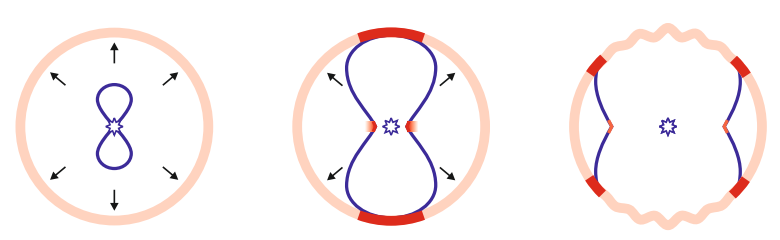

Fig. 2. Schematic representation of the interaction of the hour glass shaped BSG shell (blue) with the RSG shell (orange). The collision regions are marked with red color; they form the brightest parts of the nebula.

\section{Discussion}

Figure 2 illustrates a simplified picture of the formation of multiple ring nebulae, according to our model. It contains two kinematic components: a stationary, spherical RSG shell and an expanding hour glass structure. The strongly emitting parts of the structure are the collision surfaces, which are marked in red in the figure. We believe that both kinematic components occur in the circumstellar medium of massive stars. RSG shells are unambiguously predicted (García-Segura 2007), although they have not yet been confirmed observationaly. Expanding hour glass 
structures, on the other hand, are a well documented feature in circumstellar nebulae of low and high mass stars (see Nota et al. 1995; Langer et al. 1999) and are thought to be confined by a circumstellar disk in the equatorial plane of the central star.

A number of predictions emerge from this simple model. First, the collision starts about $10^{4} \mathrm{yr}$ after the onset of the blue loop. This timing is set by the expansion speed of the BSG shell and the radius of the RSG shell. Second, the lifetime of the nebula is determined by the duration of the collision phase, as the emission timescale is shorter than that. In our example, this is about $10^{4} \mathrm{yr}$, or about $1 \%$ of the core helium burning lifetime. This provides an upper limit to the expected number of multiple ring nebulae. Third, the rotation rate of the central star during the collision is high for a BSG, since it almost recovers from critical rotation. At the time of maximum brightness of the nebula, the equatorial rotation rate of our central star is about $80 \mathrm{~km} \mathrm{~s}^{-1}$ (Fig. A.2). Fourth, as all the material in the nebula is ejected after the first dredge-up phase of the central star, the nebula material is nitrogen-rich, enhanced here by a factor of 6.5, and carbon and oxygen depleted by factors of 6.5 and 1.5 , respectively. We note that the level of $N$-enrichment predicted by current stellar evolution models is quite uncertain (see Hunter et al. 2008), but an RSG phase is expected to produce some nitrogen enhancement. Due to the assumptions of efficient rotational mixing (Heger \& Langer 2000), the star and nebula in our model are more enriched than expected from non-rotating stellar models. Fifth, one ingredient of our simple model, namely the RSG shell, is expected for massive stars, but not so for low mass stars which produce planetary nebulae. Therefore, while analogous expanding hourglass structures are observed for both cases (Langer 2000), the multiple ring nebulae formed through the collision process shown in Fig. 2 are expected around massive stars, but not as planetary nebulae. In this sense, the polar caps observed around the blue supergiant Sher 25 might be considered as the first indirect empirical confirmation of an RSG shell.

Previous models of multiple ring nebulae were mostly constructed in the context of the triple-ring structure observed around SN 1987A (Burrows et al. 1995; Crotts \& Heathcote 2000). While single star models often fail to explain important features (e.g., Martin \& Arnett 1995; Meyer 1997; Woosley et al. 1997), many invoke rather complex binary phenomena (e.g., Podsiadlowski et al. 1991; Blondin \& Lundqvist 1993; Llyod et al. 1995; Morris \& Podsiadlowski 2005). While we do not attempt to reproduce the circumstellar medium of SN 1987A, a single star approach with suitable choices for the major parameters in our model (initial mass, initial rotation rate, metallicity) appears promising and will be pursued in the near future. The current failure of single star models to produce suitable blue loops and blue supergiant pre-supernova models may have to do more with missing physics in stellar evolution models rather than supporting the evidence for a binary progenitor of SN 1987A (Woosley et al. 1997).

Various multiple ring nebulae around blue supergiants have been observed in the last 20 years (Smith et al. 2007). While our generic numerical model was not designed to correspond to any of them, many of the general properties of these nebulae are well reproduced. Most striking is the agreement of the emission geometries. While the nebula around the B1.5 Ia supergiant Sher 25 shows two polar caps and one equatorial ring (Brandner et al. 1997) and the other objects discussed by Smith et al. (2007) rather show narrow rings, including the "twin" of the SN 1987A nebula around HD 168625 (Smith 2007), all these structures occur as an evolutionary sequence in our model. Expansion velocities of the inner ring $\left(\sim 18 \mathrm{~km} \mathrm{~s}^{-1}\right)$ and the outer collision products $\left(\sim 50 \mathrm{~km} \mathrm{~s}^{-1}\right)$, the spatial scale of about $1 \mathrm{pc}$, and the kinematic nebula age agree rather well with empirical values. The rotation velocity of our stellar model fits well to the derived value of $\sim 70 \mathrm{~km} \mathrm{~s}^{-1}$ for Sher 25 (Hendry et al. 2008). The central star and nebula of our model are nitrogen enriched, as are most of the observed nebulae.

We note that the emission in our model is caused by compressional heating, which may be in conflict with evidence for photoionization being the dominant process in some observed multiple ring nebulae (see Smith et al. 2007). And indeed, looking at the density distributions shown in Fig. A.4, which might resemble emission geometries in the pure photo-ionization case, the situation appears more complex. In our simulation, the thick RSG shell $(\Delta r \simeq r \simeq 1 \mathrm{pc}$ ) collapses in two parts (at $r \simeq 1.2 \mathrm{pc}$ and $r \simeq 1.7$ ) due to a cooling instability. However, this collapse is questionable since it requires a long timescale - our shell has an age of close to $10^{6} \mathrm{yr}$, while in many cases the shell will be much younger at the time of collision - and since the employed cooling function is uncertain for temperatures below $10000 \mathrm{~K}$. Without this collapse, its density would only be about $2 \times 10^{-25} \mathrm{~g} \mathrm{~cm}^{-3}$ (or 0.1 particles $/ \mathrm{cm}^{3}$ ), which may render it unobservable even if it were photoionized. However, even in the case of the collapsed RSG shell as in our simulation, the collision leads to a clear density enhancement. In panel 2 of Fig. A.4, we see that in our model, the density enhancement in the polar caps is about a factor 5. This is to be considered a lower limit, as higher resolution models might approach the theoretically expected enhancement factor of about 100, which follows from the (well realized) isothermal shock approximation and a Mach number of about 10. The lower panels of Fig. A.4 show that in order to represent the rings of SN 1987A, further refinements are required, which, might be achieved by altering the properties of the RSG shell. For this particular case, this may indeed be justified, as the lifetime of the final RSG stage of the progenitor of SN 1987A might have been quite short (Woosley 1988; Langer 1991a).

Even though our model does not fit any of the observed cases in detail, the approximate agreement with most general properties of this class of objects encourages us to produce tailored models for individual nebulae as next step. Our results indicate that stars with multiple ring nebulae might have just left the RSG branch - as stellar evolution models show for the case of SN 1987A (Woosley 1988; Langer 1991a) and furthermore, that binarity may not be required to obtain multiple ring emission geometries.

Acknowledgements. We are grateful to the anonymous referee for helpful remarks which lead to significant improvements of this paper. We would like to thank Karolien Lefever for providing us with a grid of atmospheric models for hot stars and to thank Anthony Marston, Nathan Smith and Bob van Veelen for helpful discussions. A. J. V. M. acknowledges support from NFS grant AST0507581. AH was supported by the DOE Program for Scientific Discovery through Advanced Computing (SciDAC; grants DOEFC02-01ER41176 and DOE-FC02-06ER41438) and performed this work under the auspices of the National Nuclear Security Administration of the US Department of Energy at Los Alamos National Laboratory under Contract No. DE-AC52-06NA25396. This work was supported by the Dutch Stichting Nationale Computerfaciliteiten (NCF).

\section{References}

Bjorkman, J. E., \& Casinelli, J. P. 1993, ApJ, 409, 429 Blondin, J. M., \& Lundqvist, P. 1993, ApJ, 405, 337

Brandner, W., Grebel, E. K., Chu, Y. H., \& Weis, K. 1997, ApJ, 475, L45

Burrows, C. J., Krist, J., Hester, J., Jeff, et al. 1995, ApJ, 452, 680

Clark, D. A. 1996, ApJ, 457, 291

Crotts, A. P. S., \& Heathcote, S. R. 2000, ApJ, 528, 426 
Eldridge, J. J., Genet, F., Daigen, F., \& Mochkovitch, R. 2005, MNRAS, 367, 186

García-Segura, G. 2007, JKAS, 40, 147

García-Segura, G., \& Franco, J. 1996, ApJ, 469, 171

García-Segura, G., Mac Low, M. M., \& Langer, N. 1996a, A\&A, 305, 229

García-Segura, G., Langer, N., \& Mac Low, M. M. 1996b, A\&A, 316, 133

García-Segura,G., Langer, N., Różyczka, M., \& Franco, J. 1999, ApJ, 517, 767

Heger, A., \& Langer, N. 1998, A\&A, 334, 210

Heger, A., \& Langer, N. 2000, ApJ, 544, 1016

Heger, A., Langer, N., \& Woosley, S. E. 2000, ApJ, 528, 368

Hendry, M. A., Smartt, S. J., Skillman, E. D., et al. 2008, submitted [arXiv:0803.4262]

Hunter, I., Brott, I., Lennon, D. J., et al. 2008, ApJ, 676, L29

Langer, N. 1991a, A\&A, 243, 155

Langer, N. 1991b, A\&A, 252, 669

Langer, N. 1998, A\&A, 329, 551

Langer, N. 2000, Science, 287, 2430

Langer, N., García-Segura, G., \& Mac Low, M. M. 1999, ApJ, 520, 49

Lloyd, H. M., O’Brien, T. J., \& Kahn, F. D. 1995, MNRAS, 273, L192

Lefever, K., Puls, J., \& Aerts, C. 2007, A\&A, 463, 1093

MacDonald, J., \& Bailey, M. E. 1981, MNRAS, 197, 995
Mac Low, M.-M., McCray, R., \& Norman, M. L. 1989, ApJ, 337, 141

Martin, C. J., \& Arnett, D. 1995, ApJ, 447, 378

Meyer, F. 1997, MNRAS, 285, L11

Nieuwenhuijzen, H., \& Jager, C. 1990, A\&A, 231, 134

Nota, A., Livio, M., Clampin, M., \& Schulte-Ladbeck, R. 1995, ApJ, 448, 788

Owocki, S., Cranmer, S. R., \& Gayley, K. G. 1996, ApJ, 472, L115

Podsiadlowski, P., Fabian, A. C., \& Stevens, I. R. 1991, Nature, 354, 43

Podsiadlowski, P., \& Morris, T. S. 2006, Stars with B[e] Phenomenon, ed. M.

Kraus, \& A. S .Miroshnichenko, ASP Conf. Ser., 355, 259

Puls, J., Urbaneja, M. A., Venero, R., et al. 2005, A\&A, 435, 669

Smith, N. 2007, AJ, 133, 1034

Smith, N., Bally, J., \& Walawender, J. 2007, AJ, 134, 846

Stone, J. M., \& Norman, M. L. 1992, ApJS, 80, 753

van Marle, A. J., Langer, N., \& García-Segura, G. 2005, A\&A, 444, 837

van Marle, A. J., Langer, N., \& García-Segura, G. 2007, A\&A, 469, 941

van Marle, A. J., Langer, N., Yoon, S.-C., \& García-Segura, G. 2008, A\&A, 478, 769

Woosley, S. E. 1988, ApJ, 330, 218

Woosley, S. E., Heger, A., Weaver, T. A., \& Langer, N. 1997

[arXiv:astro-ph/9705146] 


\section{Appendix A: Additional information}

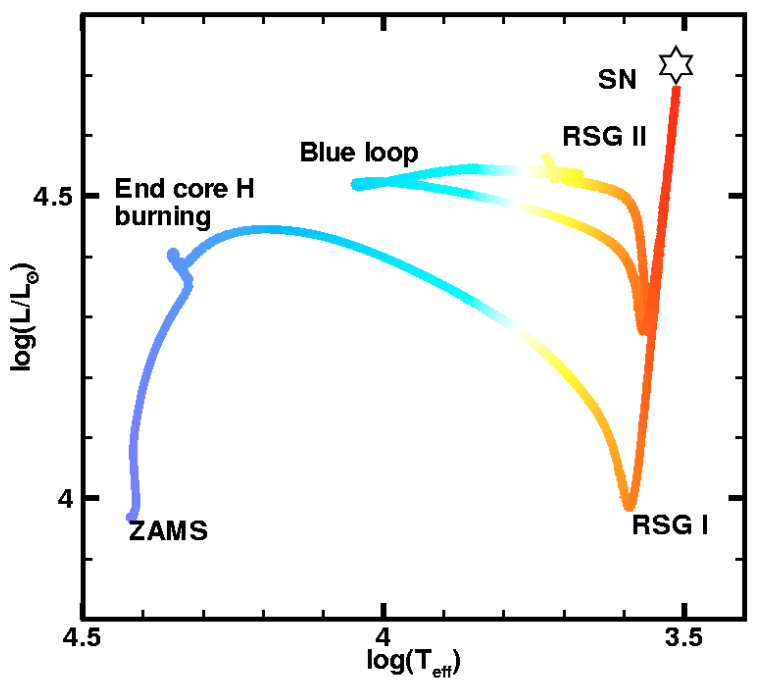

Fig. A.1. Evolution of our rotating $12 M_{\odot}$ stellar evolution model in the Hertzsprung-Russell diagram, from the zero age main sequence to the pre-supernova stage. The initial metallicity of the model is $Z=0.02$, and the initial equatorial rotation velocity is $328 \mathrm{~km} \mathrm{~s}^{-1}$.
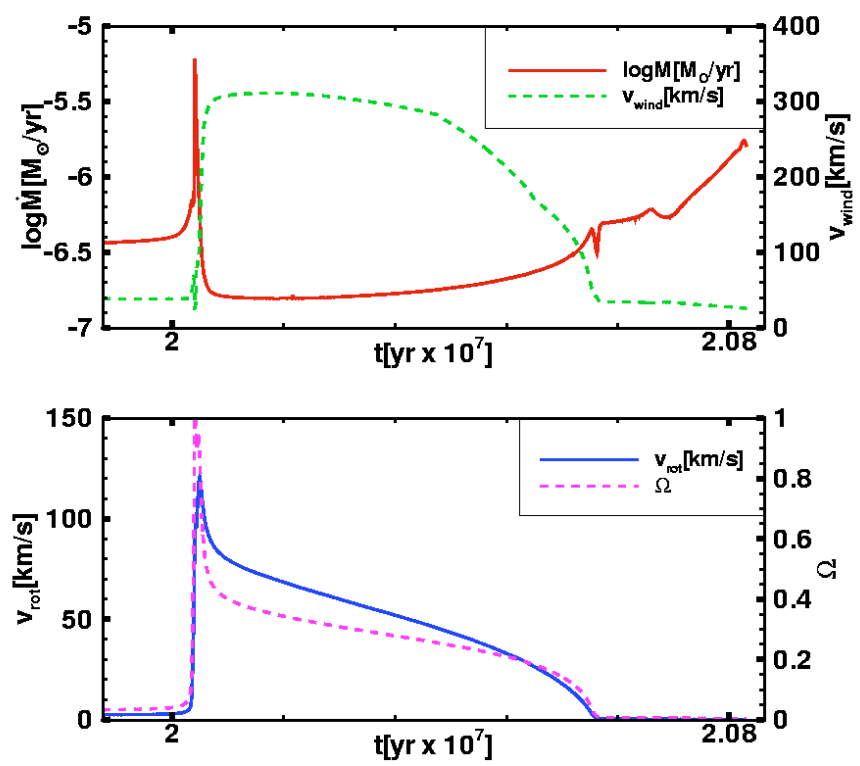

Fig. A.2. Time dependence of various quantities of the employed $12 M_{\odot}$ model, for about the final one million before core collapse. The upper panel shows stellar mass loss rate and terminal wind velocity. The lower panel depicts stellar rotational velocity, and the ratio of stellar and critical rotation rate, $\Omega$.

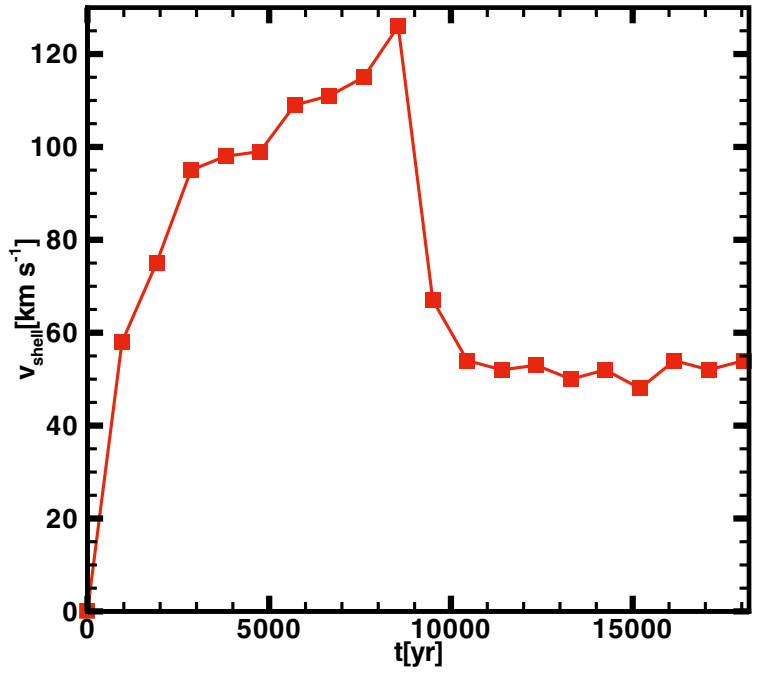

Fig. A.3. Blue supergiant shell velocity, covering a timespan of $18000 \mathrm{yr}$, from its formation $(t=0)$, until its collision with the red supergiant shell. The time between two squares corresponds to $950 \mathrm{yr}$. The time zero point corresponds to $\sim 9000 \mathrm{yr}$ before the time of the first snapshot in Fig. 1. 
S. M. Chiţă et al.: Multiple ring nebulae around blue supergiants, Online Material $p 2$
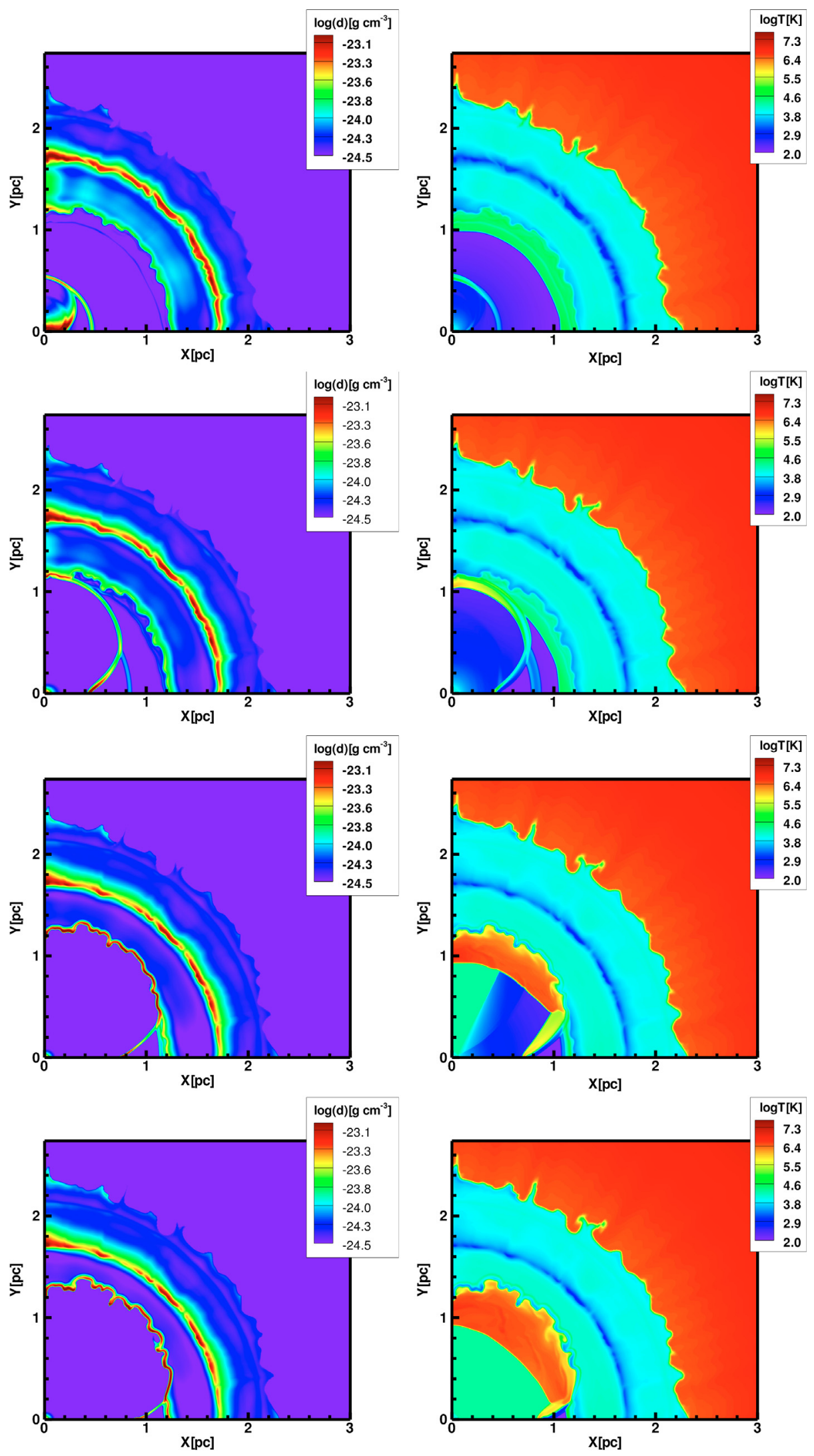

Fig. A.4. Snapshots of the density (left figures) and temperature (right figures) distributions of the circumstellar material around our $12 M_{\odot}$ model after its first red supergiant stage. The first panel corresponds to beginning of the blue supergiant stage. The spherical red supergiant shell is situated between $1 \mathrm{pc}$ and $2.2 \mathrm{pc}$. The hourglass shaped blue supergiant shell occupies the inner $0.4-0.5 \mathrm{pc}$. The second panel depicts the situation $4500 \mathrm{yr}$ later, when the polar parts of the BSG shell collide with the RSG shell. Panels 3 and 4 show the density and temperature distributions 10700 yr and $13600 \mathrm{yr}$ after the first time of Panel 1. 
S. M. Chiţă et al.: Multiple ring nebulae around blue supergiants, Online Material p 3

Fig. A.5. Movie description. Movie of the projections of the 3D emission obtained by assuming rotational symmetry of the $2 \mathrm{D}$ structures obtained from the hydro simulation, viewed with an inclination angle of $60^{\circ}$ (cf. Fig. 1, lower panel). It covers a time span of $17000 \mathrm{yr}$, starting at $2400 \mathrm{yr}$ after the time of the first panel of Fig. A.4. The time difference between two frames is about $475 \mathrm{yr}$. 